\title{
A New Means for Investigating 3-Manifolds
}

\author{
Vladimir Kovalevsky \\ Institute of Computer Graphics, University of Rostock \\ Albert-Einstein-Str. 21, 18051 Rostock, Germany \\ kovalev@tfh-berlin.de
}

\begin{abstract}
The paper presents a new method of investigating topological properties of three-dimensional manifolds by means of computers. Manifolds are represented as finite cell complexes. The paper contains definitions and a theorem necessary to transfer some basic knowledge of the classical topology to finite topological spaces. The method is based on subdividing the given set into blocks of simple cells in such a way, that a $k$-dimensional block be homeomorphic to a $k$-dimensional ball. The block structure is described by the data structure known as "cell list" which is generalized here for the three-dimensional case. Some experimental results are presented.
\end{abstract}

\section{Introduction}

Topological knowledge plays an important role in computer graphics and image analysis. Images may be represented in computers only as finite sets. Therefore it is usual to perform topological investigations in a Hausdorff space and then to transfer the results to finite sets. One of the aims of the present investigation is to demonstrate that topological investigations may be performed directly in finite sets on which a $\mathrm{T}_{0}$-topology is defined. Such a topological space can be represented in computers. We demonstrate here a new tool for investigating 3-manifolds by means of computers: the three-dimensional cell list. The same tool may be implemented for economically encoding and analyzing three-dimensional images, e.g. in computer tomography.

\section{State of the Art}

It is known from the topological literature that the problem of the complete classification of 3-manifolds is still unsolved while the classification of 2-manifolds is known since about hundred years [3]. In recent time some efforts have been made to use computers for investigating 3-manifolds.

In [9] the following method based on the notion of a spine was suggested. A spine [2] is some kind of a two-dimensional skeleton of the 3-manifold: if $K$ is a polyhedron, if $K$ collapses to $L[12$, p. 123], and if there is no elementary collapse

G. Borgefors, I. Nyström, and G. Sanniti di Baja (Eds.): DGCI 2000, LNCS 1953, pp. 57-68, 2000.

(C) Springer-Verlag Berlin Heidelberg 2000 
of $L$, then $L$ is a spine of $K$. A standard [2] or special [9] spine of a manifold has the same fundamental group as the manifold. In [9] the notion of complexity $k$ of a 3 -manifold was introduced. It is the number of vertices (0-cells) in the so called almost special spine of the manifold under consideration. It has been shown that the singular graph of a special spine is a regular graph of degree 4 . There are only finitely many different spines corresponding to a given regular graph of degree 4 . Thus it is possible to enumerate all spines with a given number of vertices.

Matveev has also introduced the so called T-transformation, which transforms a spine of a given manifold to another spine of the same manifold, which may be simpler. He also uses topological invariants introduced in [14]. More than 1000 3 -manifolds of complexity $k$ up to nine have been analyzed by means of this method [9].

We suggest here another method of using computers for the investigation of 3-manifolds. According to our method a 3-manifold is represented as an abstract cell complex (ACC) [6] with a minimum number of cells. It is encoded by the cell list as described in Section 5. It is easy to see that homeomorphic cell lists correspond to combinatorially homeomorphic manifolds. The question whether the minimum cell list of a 3-manifold is unique is recently open. There is the hope that in the case that it is not unique, the number of different cell lists of a 3-manifold (of a limited complexity) with a minimum number of cells is not too large, so that all such lists may be exhaustively tested by a computer whether they are combinatorially homeomorphic to a cell list of some already known manifold. In this presentation we describe our method of computing the cell list with a minimum number of cells for a given 3-manifold.

\section{Basic Notions}

It is known [10] that any 3-manifold may be triangulated and that homeomorphic 3-manifolds are combinatorially homeomorphic. Two complexes are called combinatorially homeomorphic if their simplicial schemata become isomorphic after finite sequences of elementary subdivisions [12, p. 24]. However, simplicial complexes contain too many elements and are therefore difficult to process. Simplices may be united to greater cells by an operation inverse to the subdivision: a subcomplex combinatorially homeomorphic to a $k$-simplex (or equivalently to a $k$-ball) may be declared to be a $k$-dimensional cell or a $k$-cell. In what follows we shall write "homeomorphic" for "combinatorially homeomorphic".

While simplices are mostly considered as subsets of a Euclidean space we prefer to work with ACC's [6]. An ACC is a set of abstract cells. A non-negative integer is assigned to each cell. It is called the dimension of the cell. The set is provided with an antisymmetric, irreflexive and transitive binary relation called bounding relation. If the cell $c_{1}$ bounds the cell $c_{2}$ it is usual to write $c_{1}<c_{2}$. A cell can only bound another cell of higher dimension.

ACC's differ both from simplicial and Euclidean complexes in so far that a cell is never a part of another cell. This property makes it possible to easily introduce the notion of open subsets of an ACC and thus to define a $\mathrm{T}_{0}$-topology on it in accordance 
with classical axioms [6]. Although an ACC is a quotient of some Hausdorff space we do not consider the cells as subsets of a Hausdorff space, which subsets are infinite ones and therefore not representable in computers. We rather consider cells as elements of an abstract finite set. This is another advantage of the ACC's since the topological space of a finite ACC may be directly and completely represented in a computer. Thus there is no necessity to consider theoretical problems in a Hausdorff space (which is not representable in computers) and then to transfer the results to a different set represented in the computer. This advantage of the ACC's is widely used in the present investigation.

One of our methods of representing 3-manifolds in computers consists in constructing a four-dimensional ACC in the computer, in defining a strongly connected subset of the ACC and in calculating the boundary of the subset.

To make the number of cells as little as possible we subdivide the ACC $A$ representing a 3-manifold into subsets each of which is homeomorphic to an open $k$-ball. We call such a subset a $k$-dimensional block cell or a $k$-block of $A$. A block $b_{1}$ of $A$ is said to bound another block $b_{2}$ of $A$ if $b_{1}$ contains a cell of $A$ which bounds another cell of $A$ contained in $b_{2}$. In this way a bounding relation is defined on the set of blocks of $A$ and the set becomes an ACC $B$ called the block complex of $A$. The blocks are cells of $B$. The topology of the block complex $B$ is a quotient topology of that of the underlying $\mathrm{ACC} A$, however, there is no necessity to consider it as a quotient topology of an Euclidean space.

\section{Incidence Structures}

\subsection{The Main Idea}

In topological literature manifolds are often represented as cell complexes. Thus e.g. the surface of a torus may be represented as a complex consisting of a 0-cell, two 1-cells and one 2-cell (Fig. 1a). This representation has the advantage of being very simple.

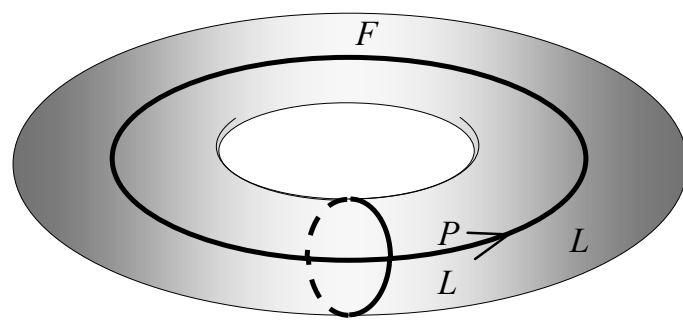

a

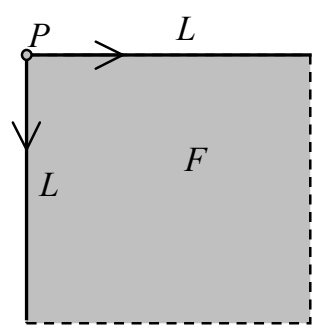

$b$

Fig. 1. Representations of the surface of a torus (a) and of a simple complex (b) 
However, if one would try to interpret this representation as an ACC, difficulties would occur since e.g. the ACC's corresponding to Fig. 1a and Fig. 1b are the same: the same sets of four cells, the same bounding relation and the same dimensions of the cells. The difference between these two complexes is that each of the 1-cells in Fig. 1a bounds the 2-cell two times, on both sides. This may be seen, if one considers the embedding of the complex in an Euclidean space: a neighborhood of a point on the 1-cell contains two half-disks each of which lies in one and the same 2-cell. However, there is no possibility to describe this relation in the language of the ACC's.

Since one of our aims is to consider a purely combinatorial approach with no relation to a Euclidean space we consider the possibility to overcome this difficulty by introducing the notion of an incidence structure.

Definition PB: A $k$-block is called proper if its closure is homeomorphic to a closed $k$-ball. A block complex is called proper if all its blocks are proper.

Thus when considering Fig. 1a as a representation of a block complex then it is not a proper one: though each $k$-block is homeomorphic to an open $k$-ball the closures of the blocks are not homeomorphic to closed $k$-balls.

An example of a proper block complex for the surface of a torus $(n=2)$ is shown in Fig. 2. The only drawback of this representation is that it has too many blocks as compared to Fig. 1a.

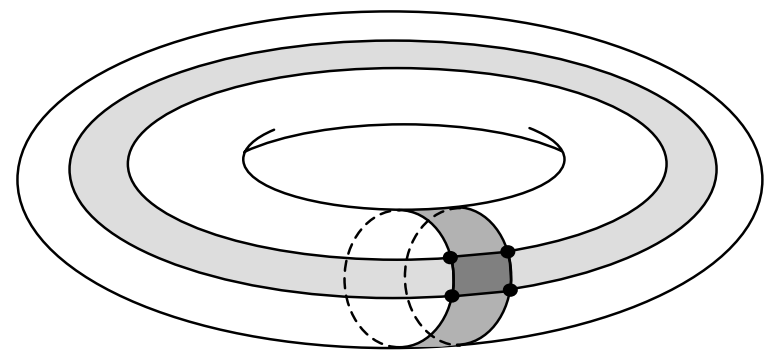

Fig. 2. A proper block complex of the surface of a torus

It is possible to reduce the number of blocks of a proper block complex while uniting two adjacent $k$-blocks which are not mutually simple (see Section 6 for the definition of "simple") by an operation inverse to the elementary subdivision. It may happen, that each of the united blocks was incident to one and the same third block. Then the union of this two blocks would be incident to the third block twice, at two different locations. In such a case we can loose some information about the topological structure of the set of blocks incident with the united block since the description of a block complex as an ACC cannot indicate that a block is multiply incident with one and the same other block.

To overcome this drawback we introduce for each block the so called incidence structure.

Definition IN: Two cells of an ACC are called incident with each other if either they are identical, or one of them bounds another one.

Definition IS: The incidence structure of a block $B C$ of a proper block complex $K$ is a subcomplex of $K$ containing all blocks incident with $B C$ except $B C$ itself.

This subcomplex may be described as an ACC: by the set of its blocks, each block represented by its label, and the bounding relation. 
To preserve the topological information about the set of blocks incident with a given block $B C$ the incidence structure of $B C$ must be stored before the uniting of blocks. During the uniting of two blocks the label of one of them in the incidence structure must be replaced by the label of another one. In this way it becomes possible that the label of one and the same block multiply occurs in the incidence structure of another block.

The incidence structures of all blocks of a block complex must be stored in a data structure which is a generalization of the cell list [6]. The former cell list was designed to describe two-dimensional Cartesian ACC's [7,8] where a point may be incident with at most four lines. In the generalized three-dimensional cell list the number of blocks incident with a point or with a curve in $3 \mathrm{D}$ is not limited. This property is important for transformations of block complexes during topological investigations.

\subsection{Incidence Structures in Multidimensional Spaces}

We will show in what follows that the incidence structure of any block of a multidimensional block complex representing a closed manifold is similar to the union of two topological spheres. This fact is the basis of the development of data structures enabling an economical representation of multidimensional block complexes in computers. To prove the necessary theorem we remain the reader some definitions.

An ACC $A$ is an Alexandroff space $[1,5]$ and hence there exists in $A$ the smallest open neighborhood of each cell $c \in A$. It is the set containing $c$ and all cells of $A$, bounded by $c$. We denote it by $\operatorname{SON}(c, A)$. For the incidence structure we need the subcomplex $\operatorname{SON}(c, A)$ without the cell $c$ itself: we denote it by $\operatorname{SON}^{*}(c, A)=$ $=\mathrm{SON}(c, A)-\{c\}$.

The closure $\mathrm{Cl}(c, A)$ is a notion dual to $\operatorname{SON}(c, A)$. It is the set containing $c$ and all cells of $A$, bounding $c$. Again, we need the set without $c$ itself: $\mathrm{Cl}^{*}(c, A)=$ $=\mathrm{Cl}(c, A)-\{c\}$. The incidence structure of a proper block $B C$ is the union:

$$
I S(B C, A)=\mathrm{SON}^{*}(B C, A) \cup \mathrm{Cl}^{*}(B C, A) .
$$

The incidence structure of a non-proper block containing fewer cells must be computed while starting with that of the original proper block complex and uniting some blocks which are not mutually simple.

Definition BI: An isomorphism between two complexes, which retains the bounding relation, is called $B$-isomorphism. $B I: A \rightarrow B$ is a $\mathrm{B}$-isomorphism iff for any $a_{1}, a_{2} \in A, a_{1}<a_{2}$ implies $B I\left(a_{1}\right)<B I\left(a_{2}\right)$.

Theorem SN: The set $\operatorname{SON}^{*}\left(c^{k}, M^{n}\right)$ of any $k$-cell $c^{k}$ of an $n$-manifold $M^{n}$ is B-isomorphic to an $(n-k-1)$-dimensional sphere if $c^{k}$ does not belong to the boundary $\partial M^{n}$ and if $0 \leq k \leq n-1$. The set $\mathrm{Cl}^{*}\left(c^{k}, M^{n}\right)$ is then B-isomorphic to an $(k-1)$ dimensional sphere.

To prove the Theorem we prove at first the particular case of $k=0$, which is the contents of the following

Lemma: The set $\mathrm{SON}^{*}\left(c^{0}, M^{n}\right)$ of a 0 -cell $c^{0} \in M^{n}$ is B-isomorphic to an (n-1)dimensional sphere. 
Proof of Lemma: According to the definition of an $n$-manifold $M^{n}$ the SON of a point (i.e. of a 0 -cell) $c^{0} \in M^{n}$ is an open $n$-ball $\mathrm{B}^{n}$. The frontier of $\mathrm{B}^{n}$ is an $(n-1)$ sphere $\mathrm{S}^{(n-1)}$. Consider the set $S=\mathrm{SON}^{*}\left(c^{0}, M^{n}\right)$. Each cell $c^{k} \in S$ has some cells in $\mathrm{S}^{(n-1)}$ which bound $c^{k}$. Let us join them to a $(k-1)$-dimensional block $b^{(k-1)}$ : a block $b^{(k-1)}$ corresponding to $c^{k}$ is a subset of $\mathrm{S}^{(n-1)}$ homeomorphic to an open $(k-1)$-dimensional ball, containing all $(k-1)$-dimensional cells $c^{(k-1)} \in \mathrm{S}^{(n-1)}$ which bound $c^{k}$ and containing also the closures of all cells of dimension $k-2$ each of which bounds at least two of the $c^{(k-1)}$. With other words:

$$
b^{(k-1)}\left(c^{k}\right)=U\left(c^{k}\right)-\partial U\left(c^{k}\right), \quad \text { where } U\left(c^{k}\right)=\mathrm{Cl}^{*}\left(c^{k}\right) \cap \mathrm{S}^{(n-1)}
$$

All such blocks compose an (n-1)-dimensional block complex $S B^{(n-1)}$ of $\mathrm{S}^{(n-1)}$, homeomorphic to $\mathrm{S}^{(n-1)}$ and thus being a topological $(n-1)$-sphere. The map I: $S \rightarrow S B^{(n-1)}$ takes each $k$-dimensional cell $c^{k} \in S$ to a $(k-1)$-dimensional block of $S B^{(n-1)}$ corresponding to $c^{k}$. Under rather general suppositions about $M^{n}$ the map $I$ retains the bounding relation: for any two cells $a, b \in S, a<b$ implies $I(a)<I(b)$. Thus $I$ is a B-isomorphism.

Proof of the Theorem: Consider the SON of a 0 -cell $c^{0}$ and a $k$-cell $c^{k} \in \operatorname{SON}^{*}\left(c^{0}, M^{n}\right), 1 \leq k \leq n-1$. According to Lemma $c^{k}$ will be mapped (as an element of $\left.\operatorname{SON}^{*}\left(c^{0}, M^{n}\right)\right)$ by $I$ onto a $(k-1)$-dimensional cell $a^{(k-1)}$ of an $(n-1)$-dimensional sphere $\mathrm{S}^{(n-1)}$. Suppose, the Theorem is true for a $(k-1)$-dimensional cell of a manifold. Since $\mathrm{S}^{(n-1)}$ is a manifold, $\mathrm{SON}^{*}\left(a^{(k-1)}, \mathrm{S}^{(n-1)}\right)$ must be B-isomorphic to a sphere of the dimension:

$$
(n-1)-(k-1)-1=n-1-k+1-1=n-k-1 .
$$

However, $I$ maps $\mathrm{SON}^{*}\left(c^{k}, M^{n}\right)$ onto $\mathrm{SON}^{*}\left(a^{(k-1)}, \mathrm{S}^{(n-1)}\right)$ and the letter onto $\mathrm{S}^{(n-k-1)}$. Thus, if the Theorem is true for a $(k-1)$-dimensional cell it is also true for a $k$-dimensional one. According to Lemma the Theorem is true for $k=1$. Therefore, it is true for any $1 \leq k \leq(n-1)$.

To prove the assertion concerning $\mathrm{Cl}^{*}$ it is sufficient to consider a set dual to $M^{n}$, where each $k$-cell is replaced by an $(n-k)$-cell, the bounding relation is reversed and the SON of a cell $c$ is replaced by its closure.

Fig. 3 shows the $\mathrm{SON}^{*}$ of a point in a 3D Cartesian ACC and its B-isomorphic map onto the surface of an octahedron, which surface is a $\mathrm{S}^{2}$. The SON* of a point contains 8 cubes $V_{1}$ to $V_{8}$ ( $V_{2}$ is removed), 12 faces and 6 edges. The B-isomorphic surface of an octahedron contains 8 faces, 12 edges and 6 points.

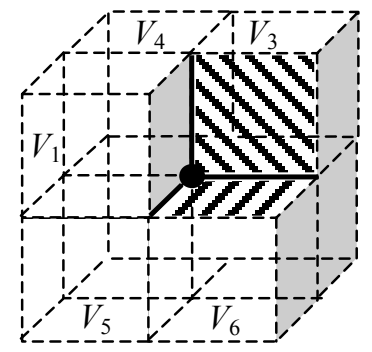

a

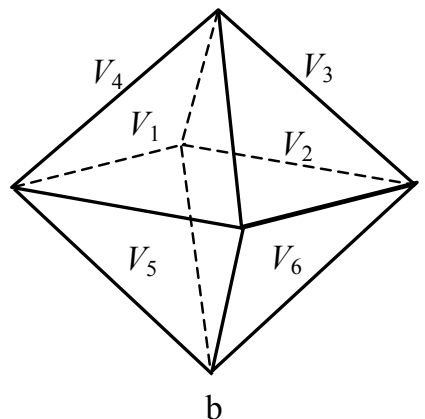

$\mathrm{b}$

Fig. 3. The SON of a point (a) and the B-isomorphic surface of an octahedron (b) 
The above results are illustrated in Table 1 showing the incidence structures of interior cells (or blocks) of a three-dimensional Cartesian ACC $A^{3}$. In cases of spaces of dimension 2 and 3 the union of $\mathrm{SON}^{*}$ with $\mathrm{Cl}^{*}$ happens to be $\mathrm{B}$-isomorphic to a two-dimensional sphere for cells of any dimension. It should be noted that this fact is of no importance for applications since the implementation of a data structure isomorphic to the union $S^{1} \cup S^{0}$ is simpler then that of $S^{2}$. Table 1 shows the incidence structures of cells $c^{k}$ of all dimensions $k$ and the 2-spheres B-isomorphic to them.

We use the incidence structures to describe non-proper block complexes. Such a description is the list of incidence structures of all blocks of a complex, called the cell list $[6,7]$. The cell list for 3D complexes is described below in Section 5. According to Theorem SN the incidence structures of a $k$-block in an $n$-dimensional manifold $M^{n}$ consists of two complexes one of which is B-isomorphic to $\mathrm{S}^{(n-k-1)}$ and the other to $\mathrm{S}^{(k-1)}$. Thus the topological structure of $M^{n}$ may be described as a list of descriptions isomorphic to spheres of lower dimensions. Therefore it may be recursively composed of structures isomorphic to $S^{0}$ and $S^{1}$ which are a pair of points and a cyclically closed sequence respectively.

Table 1. Incidence structures in a 3D space

\begin{tabular}{|c|c|c|c|c|c|}
\hline & \multicolumn{2}{|c|}{$\mathrm{Cl} *\left(c^{k}, A^{3}\right)$} & \multicolumn{2}{|c|}{$\mathrm{SON}^{*}\left(c^{k}, A^{3}\right)$} & \multirow{3}{*}{$\begin{array}{l}\mathrm{Cl}^{*} \cup \mathrm{SON}^{*} \\
\text { min. sphere }\end{array}$} \\
\hline dimension $k$ & complex & min. sphere & complex & min. sphere & \\
\hline 1 & 2 & 3 & 4 & 5 & \\
\hline 0 & $\varnothing$ & $\varnothing$ & $\operatorname{dim}=3$ & $\operatorname{dim}=2$ & \\
\hline 1 & $\operatorname{dim}=0$ & $\operatorname{dim}=0$ & $\operatorname{dim}=3$ & $\operatorname{dim}=1$ & $\operatorname{dim}=2$ \\
\hline 2 & $\operatorname{dim}=1$ & $\operatorname{dim}=1$ & $\operatorname{dim}=3$ & $\begin{array}{c}\operatorname{dim}=0 \\
\bullet\end{array}$ & $\operatorname{dim}=2$ \\
\hline 3 & $\operatorname{dim}=2$ & $\operatorname{dim}=2$ & $\varnothing$ & $\varnothing$ & $\operatorname{dim}=2$ \\
\hline
\end{tabular}

\section{The Three-Dimensional Cell List}

On the base of Theorem SN it becomes possible to construct the three-dimensional cell list as a set of tables while each line of a table describes the incidence structure of 
a block of the block complex of a 3-manifold. Each incidence structure is described as one or two ACC's each of which is B-isomorphic to a $k$-sphere with $k \leq 2$.

Let us demonstrate an example. The tables below are constituents of the 3-dimesnional cell list of the 3-manifold with boundary shown in Fig. 4.

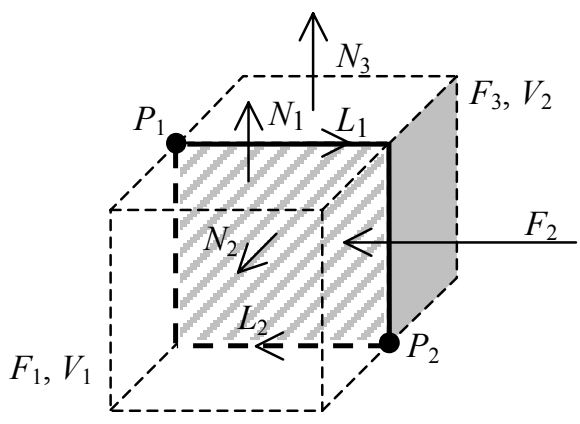

Fig. 4. Example of a simple 3-manifold with boundary

Table 2. List of the branch points (0-blocks)

\begin{tabular}{|c|c|c|c|c|c|}
\hline Label & $N_{\text {lin }}$ & Line & $N_{\text {SON }}$ & Pointer & Chained list \\
\hline \multirow{2}{*}{$P_{1}$} & \multirow{2}{*}{2} & $-L_{1}$ & 5 & $Z_{1} \rightarrow$ & $-F_{1} \rightarrow V_{1} \rightarrow-F_{2} \rightarrow V_{2} \rightarrow+F_{3} \rightarrow 0$ \\
\cline { 3 - 6 } & $+L_{2}$ & 5 & $Z_{2} \rightarrow$ & $-F_{3} \rightarrow V_{2} \rightarrow+F_{2} \rightarrow V_{1} \rightarrow+F_{1} \rightarrow 0$ \\
\hline \multirow{2}{*}{$P_{2}$} & \multirow{2}{*}{2} & $+L_{1}$ & 5 & $Z_{3} \rightarrow$ & $-F_{1} \rightarrow V_{1} \rightarrow-F_{2} \rightarrow V_{2} \rightarrow+F_{3} \rightarrow 0$ \\
\cline { 3 - 6 } & & $-L_{2}$ & 5 & $Z_{4} \rightarrow$ & $-F_{3} \rightarrow V_{2} \rightarrow+F_{2} \rightarrow V_{1} \rightarrow+F_{1} \rightarrow 0$ \\
\hline
\end{tabular}

The value $N_{\text {lin }}$ denotes the number of lines (1-blocks) incident with $P_{j}, j=1,2$, while the value $N_{\mathrm{SON}}$ denotes the number of blocks in the $\mathrm{SON}^{*}$ of the corresponding line $L_{i}, i=1,2$. These blocks compose the chained list where they are represented in the order of the rotation around $L_{i}$ corresponding to the right screw. The sign "-" before the label of an oriented face $F_{k}$ shows that the normal to $F_{k}$ points against the rotation. The pointer $Z_{m}$ points to the first element of the chained list. A zero symbol at the end of the chained list denotes that the list is not cyclically closed which may be the case for manifolds with boundary.

The list of the lines (1-blocks) contains two special blocks: the starting and the end point of the line $L_{i}$ and besides that the cyclic sequence of faces and volumes incident with $L_{i}$ as described above. The value $N_{\mathrm{SON}}$ denotes the number of blocks in the SON* of $L_{i}$.

Table 3. List of the lines (1-blocks)

\begin{tabular}{|c|c|c|c|c|c|}
\hline Label & Start & End & $N_{\text {SON }}$ & Pointer & Chained list \\
\hline$L_{1}$ & $P_{1}$ & $P_{2}$ & 5 & $Z_{5} \rightarrow$ & $-F_{1} \rightarrow V_{1} \rightarrow-F_{2} \rightarrow V_{2} \rightarrow+F_{3} \rightarrow 0$ \\
\hline$L_{2}$ & $P_{2}$ & $P_{1}$ & 5 & $Z_{6} \rightarrow$ & $-F_{3} \rightarrow V_{2} \rightarrow+F_{2} \rightarrow V_{1} \rightarrow+F_{1} \rightarrow 0$ \\
\hline
\end{tabular}


The list of faces (2-blocks) has a similar structure: the incidence structure of a face $F_{i}$ contains two special blocks (these are now the two incident 3-blocks) and a cyclic sequence of $N_{C l}$ blocks in $\mathrm{Cl}^{*}\left(F_{i}\right)$. The first symbol is repeated at the end of the chained list to show that the sequence is closed.

Table 4. List of faces (2-blocks)

\begin{tabular}{|c|c|c|c|c|c|}
\hline Label & + Vol & - Vol & $N_{C l}$ & Pointer & Chained list \\
\hline$F_{1}$ & - & $V_{1}$ & 4 & $Z_{7} \rightarrow$ & $P_{1} \rightarrow-L_{2} \rightarrow P_{2} \rightarrow-L_{1} \rightarrow P_{1}$ \\
\hline$F_{2}$ & $V_{1}$ & $V_{2}$ & 4 & $Z_{8} \rightarrow$ & $P_{1} \rightarrow-L_{2} \rightarrow P_{2} \rightarrow-L_{1} \rightarrow P_{1}$ \\
\hline$F_{3}$ & - & $V_{2}$ & 4 & $Z_{9} \rightarrow$ & $P_{1} \rightarrow+L_{1} \rightarrow P_{2} \rightarrow+L_{2} \rightarrow P_{1}$ \\
\hline
\end{tabular}

Table 5. List of volumes (3-blocks)

\begin{tabular}{|c|c|c|c|c|c|}
\hline Label & $N_{f}$ & Face & $N_{\mathrm{Cl}}$ & Pointer & Chained list \\
\hline \multirow{3}{*}{$V_{1}$} & \multirow{3}{*}{2} & $+F_{1}$ & 4 & $Z_{10} \rightarrow$ & $P_{1} \rightarrow-L_{2} \rightarrow P_{2} \rightarrow-L_{1} \rightarrow P_{1}$ \\
\cline { 3 - 6 } & & $-F_{2}$ & 4 & $Z_{11} \rightarrow$ & $P_{1} \rightarrow+L_{1} \rightarrow P_{2} \rightarrow+L_{2} \rightarrow P_{1}$ \\
\hline \multirow{3}{*}{$V_{2}$} & \multirow{2}{*}{2} & $+F_{2}$ & 4 & $Z_{12} \rightarrow$ & $P_{1} \rightarrow-L_{2} \rightarrow P_{2} \rightarrow-L_{1} \rightarrow P_{1}$ \\
\cline { 3 - 6 } & $+F_{3}$ & 4 & $Z_{13} \rightarrow$ & $P_{1} \rightarrow+L_{1} \rightarrow P_{2} \rightarrow+L_{2} \rightarrow P_{1}$ \\
\hline
\end{tabular}

The value $N_{f}$ denotes the number of faces $F_{j}$ incident with $V_{i}, i=1,2$, while the value $N_{C l}$ denotes as before the number of blocks in $\mathrm{Cl}^{*}\left(F_{j}\right)$.

A presentation of the fundamental group of a given complex may be computed from its cell list by the method suggested by Poincaré [11] and proved by Tietze [13]. According to this method it is necessary to find the spanning tree of the 1-dimesnional skeleton of the complex and ignore all 1-cells in the tree. Each of the remaining 1 -cells is a generator, the concatenation of the generators in the perimeter of each 2-cell, being equated to identity, is a relation of the presentation of the fundamental group.

In a similar way cell lists for manifolds (with and without boundary) of greater dimension may be constructed. The list consists of incidence structures each of which is B-isomorphic to a sphere of some lower dimension and thus may be described by a cell list of lower dimension. Thus e.g. in the cell list of a 5-manifold the incidence structure of a 0 - and of a 5-block is a cell list of dimension 4 . The incidence structures of other blocks are lists of lower dimensions.

The gained understanding shows that cell lists for block complexes of manifolds of any dimension may be constructed by means of a recursion: the cell list of an $n$-manifold consists of lists of lower dimensions. 


\section{Computer Experiments}

\subsection{Generating Block Complexes and Cell Lists of 3-Manifolds}

We use two methods of producing block complexes and their cell lists in the computer. The first method implements the classical idea of gluing (or identifying) the faces of a polyhedron. The description of a polyhedron may be input into the computer manually, just in the form of a three-dimensional cell list containing a single 3-block and as many 2-blocks as the number $N_{f}$ of faces. Also a list of desired identifications of the faces and their closures (specifying the homeomorphism of the gluing) must be input. The corresponding computer program replaces the labels of some blocks and calculates the new incidence structures which are unions of the initial ones. The result is a cell list of a 3-manifold.

The second method consists in defining a strongly connected subset of a fourdimensional Cartesian ACC represented as a four-dimensional array in the computer. The boundary of the subset is then the desired 3-manifold. It is a three-dimensional ACC.

The block complex of a given ACC may be computed as follows. Consider two closed $n$-balls whose boundary intersection is an $(n-1)$-ball. Then the union of the $n$-balls is again an $n$-ball since uniting is a procedure inverse to the elementary subdivision of an $n$-cell. We call such two $n$-balls mutually simple or simple relative to each other. The union of the closures of two mutually simple $n$-cells or $n$-blocks is a closed subcomplex homeomorphic to a closed $n$-ball $\mathrm{B}^{n}$.

The program selects an arbitrary $n$-cell of the given $n$-dimensional complex as the seed of $\mathrm{B}^{n}$. Then all $n$-cells, which are simple relative to the growing ball $\mathrm{B}^{n}$, are sequentially united with it, one cell at each step. The closures of the united $n$-cells are labeled as belonging to the closure of the $n$-block. When there are no more simple cells, the rest consisting of $n$-cells which are not simple relative to $\mathrm{B}^{n}$ can be subdivided into handles of indices 0 to 2 [4, p. 28]. All $n$-cells of a handle and the cells of their closures which are not jet labeled get a label of the handle. This is accomplished in the order of decreasing indices: all handles of index 2 first, etc.

Each handle of index $k$ is then contracted to a $k$-block, i.e. a corresponding $k$-block is recorded in the cell list. The incidence structure of a block may be directly read from the closure of the $n$-cells $c^{n}$ of the corresponding handle $H$, which cells are incident with the base of $H$ : the cells of $\mathrm{Cl}^{*}\left(c^{n}\right)$ contain the labels of other handles having a common boundary with $H$, due to the labeling procedure described above.

We have developed a computer program which automatically calculates according to the described algorithm the cell list of a two- or tree-dimensional orientable manifold without boundary. The manifold must be defined as the frontier of a strongly connected subsets of a four-dimensional Cartesian ACC. The program also minimizes the number of blocks while uniting pairs of 0-blocks (points) incident with a line (1-block) until a block complex with a single 0-block, a single 3-block, $m$ 1-blocks and $m$ 2-blocks is obtained (the Euler number $N^{0}-N^{1}+N^{2}-N^{3}=1-m+m-1$ must be 0 ).

Several examples of manifolds were successfully tested. As an example we show the results of investigating a well-known 3-manifold $\mathrm{S}^{1} \times \mathrm{S}^{1} \times \mathrm{S}^{1}$ which may be obtained by identifying opposite faces of a cube. The minimized cell list of this manifold 
contains a single point, three closed curves, three faces each spanned by two curves and a single volume. Since the cell list is redundant (its redundancy is necessary for the purpose of a fast search) its contents may be represented by that of the incidence structure of the single volume. As demonstrated above, the incidence structure of any block of a 3-manifold is B-isomorphic to a 2-sphere and hence may be projected onto the plane. Fig. 5 shows a planar projection of the incidence structure of the 3-block.

In Fig. 5 elements of the same hatching represent identified blocks. The 2-blocks are denoted by $a, b$ and $c$. Primed symbols correspond to opposite orientations. The 0 and 1-dimensional blocks are denoted by combinations of the symbols of the bounded 2-blocks.

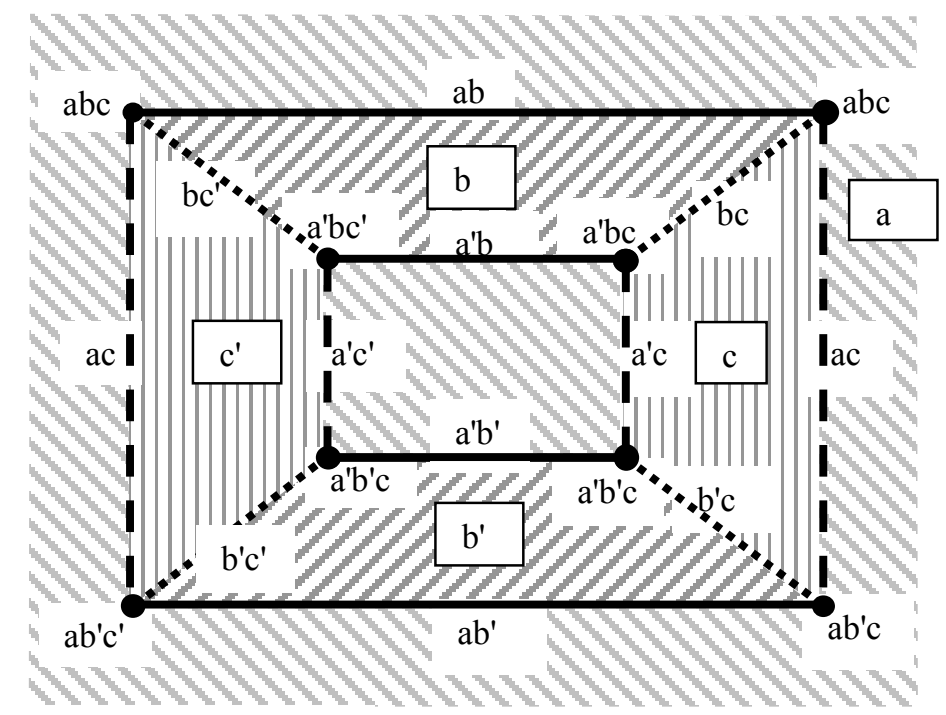

Fig. 5. Incidence structure of the 3-block of the block complex of the glued cube

A presentation of the fundamental group of this manifold may be found as follows: there is a single vertex which is the spanning tree. Therefore all three 1-blocks $a b, a c$ and $b c$ are generators. Let us denote them by $x=a b, \mathrm{y}=a c$ and $\mathrm{z}=b c$. Then the perimeter of the face $a$ (the exterior area) contains the sequence $x y x^{\prime} y^{\prime}=1$, the perimeter of the face $b$ the sequence $x z x^{\prime} z^{\prime}=1$ and the perimeter of the face $c$ the sequence $y z y^{\prime} z^{\prime}=1$. Thus the fundamental group is the free abelian group of rank 3 .

\section{Conclusion}

The described method gives the possibility to compute automatically a representation of a two- or three-dimensional manifold as a cell complex with the (almost) minimum number of cells. This evokes the hope to test by means of a fast computer the combinatorial homeomorphism of 3-manifolds as the isomorphism of cell complexes. 
The method also makes it possible to compute automatically a presentation of the fundamental group of the given manifold. The method may be useful for further investigations of 3-manifolds and may be a contribution for the solution of the still unsolved problem of classifying 3 -manifolds.

The tree-dimensional cell list developed here may be also used for economically encoding and analyzing tree-dimensional images, e.g. in computer tomography, or time sequences of two-dimensional images in digital television.

\section{References}

1. Alexandroff, P.: Diskrete Räume, Mat. Sbornik, Vol. 2 (1937) 501-518

2. Casler, B.G.: An Imbedding Theorem for Connected 3-Manifolds with Boundary, Proceedings of the American Mathematical Society, Vol. 16 (1965) 559-566

3. Dehn, M., Heegaard, P.: Analysis situs, Encyklopädie der mathematischen Wissenschaften, Vol. III, AB3, Leipzig (1907) 153-220

4. Fomenko, A.T., Matveev, S.V.: Algorithmic and Computer Methods for ThreeManifolds, Kluwer (1997)

5. Kong, T.Y., Kopperman, R., Meyer, P.R.: A topological approach to digital topology, Amer. Math. Monthly, Vol. 98 (1991) 901-917

6. Kovalevsky, V.A.: Finite Topology as Applied to Image Analysis, Computer Vision, Graphics and Image Processing, Vol. 45, No. 2 (1989) 141-161

7. Kovalevsky, V.A.: Finite Topology and Image Analysis, in Advances in Electronics and Electron Physics, P. Hawkes ed., Academic Press, Vol. 84 (1992) 197-259

8. Kovalevsky, V.A.: A New Concept for Digital Geometry, in Shape in Picture, O, Ying-Lie, Toet, A., Foster, D., Heijmans, H.J.A.M., Meer, P. (Eds.), SpringerVerlag Berlin Heidelberg (1994) 37-51

9. Matveev, S.V.: Computer classification of 3-manifolds, in Russian, TR, University of Cheliabinsk, Russia, (1999)

10. Moise, E.E.: Affine structures in 3-manifolds. V. The triangulation theorem and Hauptvermutung, Ann. Math. Vol. 56 (1952) 865-902

11. Poincaré, H.: Analysis situs, J. de l'Ècole Polyt. (2) Vol. 1 (1895) 1-123

12. Stillwell, J.: Classical Topology and Combinatorial Group Theory, Springer (1995)

13. Tietze, H.: Über die topologischen Invarianten mehrdimensionaler Mannigfaltigkeiten, Monatsh. Math. Phys. Vol. 19, (1908) 1-118

14. Turaev, V.G., Viro, O.Y.: State sum invariants of 3-manifolds and quantum 6j-symbol, Topology, Vol. 31, N 4 (1992) 865-902 